\title{
Multiple periodic solutions for second order Josephson-type differential systems
}

Shengui Zhang*

"Correspondence:
zhangshengui315@163.com
College of Mathematics and
Computer Science, Northwest
University for Nationalities, Lanzhou,
Gansu 730030, P.R. China

\begin{abstract}
In this paper, some new existence theorems are obtained for multiple periodic solutions of second order Josephson-type differential systems with partially periodic potential by using the minimax methods in critical point theory, which generalize and improve some known results in the literature.
\end{abstract}

MSC: $34 \mathrm{C} 25 ; 58 \mathrm{E} 50$

Keywords: periodic solution; critical point; second order Josephson-type differential systems; periodicity; minimax methods; the generalized saddle point theorem

\section{Introduction}

In this paper, we study the second order Josephson-type differential systems

$$
\left\{\begin{array}{l}
\ddot{u}(t)+A u(t)-\nabla F(t, u(t))=h(t), \quad \text { a.e. } t \in[0, T], \\
u(0)-u(T)=\dot{u}(0)-\dot{u}(T)=0,
\end{array}\right.
$$

where $A$ is an $(N \times N)$-symmetric matrix, $h(t) \in L^{1}\left([0, T] ; \mathbb{R}^{N}\right), T>0, \nabla F(t, x)$ denotes its gradient with respect to the second variable, that is,

$$
\nabla F(t, x)=\nabla_{x} F(t, x)=\left(\partial F / \partial x_{1}, \ldots, \partial F / \partial x_{N}\right), \quad x(t)=\left(x_{1}(t), \ldots, x_{N}(t)\right),
$$

and $F:[0, T] \times \mathbb{R}^{N} \rightarrow \mathbb{R}$ satisfies the following assumptions:

(H1) $F(t, x)$ is measurable in $t$ for each $x \in \mathbb{R}^{N}$ and continuously differentiable in $x$ for a.e. $t \in[0, T]$, and there exist $a \in C\left(\mathbb{R}^{+}, \mathbb{R}^{+}\right), b \in L^{1}\left([0, T] ; \mathbb{R}^{+}\right)$such that

$$
|F(t, x)| \leq a(|x|) b(t), \quad|\nabla F(t, x)| \leq a(|x|) b(t)
$$

for all $x \in \mathbb{R}^{N}$ and a.e. $t \in[0, T]$.

(H2) $\operatorname{dim} N(A)=m \geq 1$ and matrix $A$ has no eigenvalue of the form $k^{2} v^{2}(k \in \mathbb{N} /\{0\})$, where $v=2 \pi / T$.

(H3) There exist linearly independent vectors $e_{j} \in \mathbb{R}^{N}(1 \leq j \leq m)$ such that

$$
N(A)=\operatorname{span}\left\{e_{1}, e_{2}, \ldots, e_{m}\right\}
$$

\section{Springer}


and

$$
\int_{0}^{T}\left(h(t), e_{j}\right) d t=0
$$

This problem (1.1) occurs in various branches of mathematical physics, for example, when $A=N^{2} D^{2}$ and $-\nabla F(t, u(t))=f(u(t))=\left(a_{1} \sin u_{1}, \ldots, a_{N} \sin u_{N}\right)$, problem (1.1) reduces to the nonlinear systems of the form

$$
\left\{\begin{array}{l}
\ddot{u}(t)+N^{2} D^{2} u(t)+f(u(t))=h(t), \quad \text { a.e. } t \in[0, T], \\
u(0)-u(T)=\dot{u}(0)-\dot{u}(T)=0,
\end{array}\right.
$$

where $D$ is an $(N \times N)$-symmetric matrix. This type of problem can be applied to describe the motion of forced linearly coupled pendulums.

During the past two decades, the existence of periodic solutions for second order differential systems have been studied extensively, and many solvability conditions have been obtained via variational methods and critical point theory. In this direction we mention the papers [1-15], and we refer the reader to [16-19] for a broad introduction to variational methods and critical point theory. It might be also interesting to study the above mentioned abstract equations with more general potentials, see the paper [20].

In the classical monograph [6], Mawhin and Willem proved that problem (1.1) has at least one solution by using the saddle point theorem under the following bounded condition: there exists $g \in L^{1}\left([0, T] ; \mathbb{R}^{+}\right)$such that

$$
|F(t, x)|+|\nabla F(t, x)| \leq g(t)
$$

for all $x \in \mathbb{R}^{N}$ and a.e. $t \in[0, T]$. They obtained the following result.

Theorem A ([6]) Suppose that F satisfies (H1)-(H3), (1.3) and

(H4) there exists $T_{j}>0$ such that

$$
F\left(t, x+T_{j} e_{j}\right)=F(t, x), \quad 1 \leq j \leq m,
$$

for all $x \in \mathbb{R}^{N}$ and a.e. $t \in[0, T]$. Then Eq. (1.1) has at least one solution in $H_{T}^{1}$, where the Sobolev space $H_{T}^{1}$ is defined by

$$
\begin{gathered}
H_{T}^{1}=\left\{u:[0, T] \rightarrow \mathbb{R}^{N} \mid u\right. \text { is absolutely continuous, } \\
\left.u(0)=u(T) \text { and } \dot{u} \in L^{2}\left([0, T] ; \mathbb{R}^{N}\right)\right\}
\end{gathered}
$$

and $H_{T}^{1}$ is a Hilbert space with the norm

$$
\|u\|=\left(\int_{0}^{T}|\dot{u}(t)|^{2} d t+\int_{0}^{T}|u(t)|^{2} d t\right)^{\frac{1}{2}}, \quad u \in H_{T}^{1} .
$$

When the nonlinearity $\nabla F(t, x)$ is sublinear, that is, there exist $f, g \in L^{1}\left([0, T] ; \mathbb{R}^{+}\right)$and $\alpha \in[0,1)$ such that

$$
|\nabla F(t, x)| \leq f(t)|x|^{\alpha}+g(t)
$$


for all $x \in \mathbb{R}^{N}$ and a.e. $t \in[0, T]$, Tang $[12,13]$ researched the existence of periodic solutions for system (1.1) in the case $A \equiv 0$.

Subsequently, Feng and Han [3] generalized Theorem A to the sublinear case, and they assume that the following assumptions hold:

(H5) There exist $T_{j}>0,1 \leq r \leq m$ such that

$$
F\left(t, x+T_{j} e_{j}\right)=F(t, x), \quad 1 \leq j \leq r,
$$

for all $x \in \mathbb{R}^{N}$ and a.e. $t \in[0, T]$.

(H6) $\lim _{\|x\| \rightarrow \infty} \frac{\int_{0}^{T} F(t, x) d t}{\|x\|^{2 \alpha}}=-\infty$, as $x \in N(A) \ominus \operatorname{span}\left\{e_{1}, e_{2}, \ldots, e_{r}\right\}$.

Theorem B ([3]) Suppose that F satisfies (H1)-(H3), (H5)-(H6) and (1.4). Then Eq. (1.1) has at least $r+1$ distinct solutions in $H_{T}^{1}$.

In [15], the author obtained the following result.

Theorem C ([15]) Suppose that F satisfies (H1)-(H3), (H5), (1.4) and the following generalized Ahmad-Lazer-Paul type coercive conditions:

(H7) $\lim _{\|x\| \rightarrow \infty} \frac{\int_{0}^{T} F(t, x) d t}{\|x\|^{2 \alpha}}<-L$, as $x \in N(A) \ominus \operatorname{span}\left\{e_{1}, e_{2}, \ldots, e_{r}\right\}$, where $L$ is a positive constant. Then Eq. (1.1) has at least $r+1$ distinct solutions in $H_{T}^{1}$.

In this paper, we use a more general control function instead of $|x|^{\alpha}$ in (1.4). By using the generalized saddle point theorem due to Liu [5], we can prove the existence of multiple periodic solutions for the second order Josephson-type differential systems for a new and large range of the nonlinear term.

\section{Preliminaries}

In [6], Mawhin and Willem established a variational structure which enables us to reduce the existence of solutions for problem (1.1) to the existence of critical points of the following energy functional. Define the energy functional associated with problem (1.1) on $H_{T}^{1}$

$$
\begin{aligned}
\varphi(u)= & \frac{1}{2} \int_{0}^{T}|\dot{u}(t)|^{2} d t-\frac{1}{2} \int_{0}^{T}(A u(t), u(t)) d t+\int_{0}^{T} F(t, u(t)) d t \\
& +\int_{0}^{T}(h(t), u(t)) d t .
\end{aligned}
$$

It follows from assumption (H1) that the functional $\varphi$ is continuously differentiable. Moreover, one has

$$
\begin{aligned}
\left\langle\varphi^{\prime}(u), v\right\rangle= & \int_{0}^{T}(\dot{u}(t), \dot{v}(t)) d t-\int_{0}^{T}(A u(t), v(t)) d t+\int_{0}^{T}(\nabla F(t, u(t)), v(t)) d t \\
& +\int_{0}^{T}(h(t), v(t)) d t, \quad \forall u, v \in H_{T}^{1} .
\end{aligned}
$$

Then the solutions of problem (1.1) correspond to the critical points of $\varphi$ (see [6]). 
Let

$$
q(u)=\int_{0}^{T}\left[|\dot{u}(t)|^{2}-(A u(t), u(t))\right] d t .
$$

Therefore, we can see that

$$
\begin{aligned}
q(u) & =\|u\|^{2}-\int_{0}^{T}((A+I) u(t), u(t)) d t \\
& =\langle(I-K) u, u\rangle,
\end{aligned}
$$

where $I$ denotes the identity operator on $H_{T}^{1}$ and $K: H_{T}^{1} \rightarrow H_{T}^{1}$ is the linear self-adjoint operator defined, using Riesz representation theorem, by

$$
\int_{0}^{T}((A+I) u(t), v(t)) d t=(K u, v), \quad \forall u, v \in H_{T}^{1}
$$

It is easy to see that $K$ is compact. By classical spectral theory, we can decompose $H_{T}^{1}$ into the orthogonal sum of invariant subspaces for $I-K$

$$
H_{T}^{1}=H^{-} \oplus H^{0} \oplus H^{+},
$$

where $H^{0}=\operatorname{Ker}(I-K)=N(A)$ and $\operatorname{dim} H^{-}<+\infty$, for some $\delta>0$, we have

$$
\begin{aligned}
& q(u) \leq-\delta\|u\|^{2}, \quad \forall u \in H^{-} \\
& q(u) \geq \delta\|u\|^{2}, \quad \forall u \in H^{+} .
\end{aligned}
$$

Lemma 2.1 ([6]) There is a continuous embedding $H_{T}^{1} \hookrightarrow C\left([0, T], \mathbb{R}^{N}\right)$, and the embedding is compact. Then there exists $C_{0}>0$ such that

$$
\|u\|_{\infty}:=\max _{0 \leq t \leq T}|u(t)| \leq C_{0}\|u\|, \quad \forall u \in H_{T}^{1} .
$$

Define

$$
Y_{0}=\operatorname{span}\left\{e_{1}, e_{2}, \ldots, e_{r}\right\}, \quad Y_{1}=N(A) \ominus Y_{0}=\operatorname{span}\left\{e_{r+1}, e_{r+2}, \ldots, e_{m}\right\},
$$

then

$$
u(t)=u^{-}(t)+u^{+}(t)+P u^{0}+Q u^{0},
$$

where $u^{-} \in H^{-}, u^{+} \in H^{+}, P u^{0} \in Y_{1}$ and $Q u^{0}=\sum_{j=1}^{r} c_{j} e_{j}$. Let

$$
G=\left\{\sum_{i=1}^{r} k_{i} T_{i} e_{i} \mid k_{i} \in \mathbb{Z}, 1 \leq i \leq r\right\}
$$

be a discrete subgroup of $H_{T}^{1}$, where $\mathbb{Z}$ is the set of all integers, and let $\pi: H_{T}^{1} \rightarrow H_{T}^{1}$ /G be the canonical surjection. Let

$$
H_{T}^{1} / G=X \times V=(W \oplus Z) \times V,
$$


where $W=H^{+}, Z=H^{-} \oplus Y_{1}, V=Y_{0} / G$, then $\operatorname{dim} Z<+\infty, \operatorname{dim} V<+\infty$, and $V$ is isomorphic to the torus $T^{r}$. The element in $V$ can be represented as

$$
Q \hat{u}^{0}=\sum_{j=1}^{r} \hat{c}_{j} e_{j}
$$

where $\hat{c}_{j}=c_{j}-k_{j} T_{j}, 0 \leq \hat{c}_{j}<T_{j}$. Let

$$
\hat{u}(t)=u^{-}(t)+u^{+}(t)+P u^{0}+Q \hat{u}^{0} .
$$

By (H3) and (H5), we have

$$
\begin{aligned}
& F(t, u(t))=F\left(t, \hat{u}(t)+\sum_{i=1}^{r} k_{i} T_{i} e_{i}\right)=F(t, \hat{u}(t)), \\
& \nabla F(t, u(t))=\nabla F\left(t, \hat{u}(t)+\sum_{i=1}^{r} k_{i} T_{i} e_{i}\right)=\nabla F(t, \hat{u}(t))
\end{aligned}
$$

and

$$
\int_{0}^{T}(h(t), u(t)) d t=\int_{0}^{T}\left(h(t), \hat{u}(t)+\sum_{i=1}^{r} k_{i} T_{i} e_{i}\right) d t=\int_{0}^{T}(h(t), \hat{u}(t)) d t .
$$

Thus, $\varphi(u)=\varphi(\hat{u}), \varphi^{\prime}(u)=\varphi^{\prime}(\hat{u})$. Define $\psi: X \times V \mapsto \mathbb{R}: \psi(\pi(u))=\varphi(u)$, then $\psi$ is well defined. Moreover, $\psi$ is continuously differentiable and

$$
\psi(\pi(u))=\psi(\pi(\hat{u})), \quad \psi^{\prime}(\pi(u))=\psi^{\prime}(\pi(\hat{u})) .
$$

Definition 2.1 ([6]) Suppose that $\psi$ satisfies the (PS) condition, that is, every sequence $\left\{x_{n}\right\}$ of $X \times V$ such that $\psi\left\{x_{n}\right\}$ is bounded and $\psi^{\prime}\left\{x_{n}\right\} \rightarrow 0$ as $n \rightarrow \infty$ possesses a convergent subsequence.

Lemma 2.2 (The generalized saddle point theorem [5]) Let $X$ be a Banach space with a decomposition $X=Z+W$, where $Z$ and $W$ are two subspaces of $X$ with $\operatorname{dim} Z<+\infty$. Let $V$ be a finite-dimensional, compact $C^{2}$-manifold without boundary. Let $\psi: X \times V \rightarrow \mathbb{R}$ be a $C^{1}$-function satisfying the (PS) condition. Suppose that there exist constants $\rho>0$ and $\gamma<\beta$ such that
(a) $\inf _{x \in W \times V} \psi(x) \geq \beta$,
(b) $\sup _{x \in S \times V} \psi(x) \leq \gamma$,

where $S=\partial D, D=\{z \in Z|| z \mid \leq \rho\}$. Then the functional $\psi$ has at least cuplength $(V)+1$ critical points. 


\section{Main results}

Here are our main results.

Theorem 3.1 Suppose that assumptions (H1)-(H3), (H5) hold and there exist constants $M_{i}>0, i=0,1,2$, and a nonnegative function $\omega \in C([0, \infty),[0, \infty))$ with the properties:

$(\omega 1) \quad \omega(s) \leq \omega(t), \forall s \leq t, s, t \in[0, \infty)$;

$(\omega 2) \omega(s+t) \leq M_{0}(\omega(s)+\omega(t)), \forall s, t \in[0, \infty)$;

( $\omega 3) \quad 0 \leq \omega(s) \leq M_{1} s+M_{2}, \forall s, t \in[0, \infty)$;

$(\omega 4) \omega(s) \rightarrow+\infty$ as $s \rightarrow+\infty$.

Moreover, there exist constant $a>3$ and $f, g \in L^{1}\left([0, T] ; \mathbb{R}^{+}\right)$with

$$
\int_{0}^{T} f(t) d t<\frac{\delta}{(2+a) M_{0} M_{1} C_{0}^{2}}
$$

such that

$$
|\nabla F(t, x)| \leq f(t) \omega(|x|)+g(t)
$$

for all $x \in \mathbb{R}^{N}$ and a.e. $t \in[0, T]$, and

$$
\limsup _{|x| \rightarrow \infty} \frac{\int_{0}^{T} F(t, x) d t}{\omega^{2}(|x|)}<-\left(\frac{15}{2} \frac{1}{2 a-1}+\frac{3}{2}\right) \frac{M_{0}}{M_{1}} \int_{0}^{T} f(t) d t
$$

as $x \in N(A) \ominus \operatorname{span}\left\{e_{1}, e_{2}, \ldots, e_{r}\right\}$. Then Eq. (1.1) has at least $r+1$ distinct solutions in $H_{T}^{1}$.

Theorem 3.2 Suppose that assumptions (H1)-(H3), (H5), (3.1), (3.2) hold and

$$
\liminf _{|x| \rightarrow \infty} \frac{\int_{0}^{T} F(t, x) d t}{\omega^{2}(|x|)}>\left(\frac{15}{2} \frac{1}{2 a-1}+\frac{3}{2}\right) \frac{M_{0}}{M_{1}} \int_{0}^{T} f(t) d t
$$

as $x \in N(A) \ominus \operatorname{span}\left\{e_{1}, e_{2}, \ldots, e_{r}\right\}$. Then Eq. (1.1) has at least $r+1$ distinct solutions in $H_{T}^{1}$.

By Theorems 3.1 and 3.2, it is easy to obtain the following corollary.

Corollary 3.1 Suppose that assumptions (H1)-(H3), (H5), (3.1), (3.2) hold and

$$
\lim _{|x| \rightarrow \infty} \frac{\int_{0}^{T} F(t, x) d t}{\omega^{2}(|x|)}=+\infty \quad(\text { or }-\infty)
$$

as $x \in N(A) \ominus \operatorname{span}\left\{e_{1}, e_{2}, \ldots, e_{r}\right\}$. Then Eq. (1.1) has at least $r+1$ distinct solutions in $H_{T}^{1}$.

Remark 3.1 (i) When $A \equiv 0$, assumptions ( $\omega 1)-(\omega 4)$ and condition (3.2) were introduced in [10]. Comparing with the results in [10], the periodicity and coercivity conditions in our Theorem 3.1 are only in a part of variables of potentials, and we obtained multiplicity of periodic solutions for problem (1.1). 
(ii) To show that our Theorem 3.1 is new, we give an example to illustrate our result. For example, let $1 \leq r \leq m, x=\left(x_{1}, x_{2}, \ldots, x_{N}\right)^{T} \in \mathbb{R}^{N}$, and

$$
\begin{aligned}
F(t, x)= & \left(\frac{2}{5} T-t\right) \ln ^{2}\left[1+\left(r+1+\sum_{j=1}^{r} \sin ^{2} x_{j}+\frac{1}{2} \sum_{j=r+1}^{N} x_{j}^{2}\right)\right] \\
& +\left(T^{3}-t\right) \ln \left[1+\left(r+1+\sum_{j=1}^{r} \sin ^{2} x_{j}+\frac{1}{2} \sum_{j=r+1}^{N} x_{j}^{2}\right)\right],
\end{aligned}
$$

let $\omega(|x|)=\ln \left[1+\left(r+1+|x|^{2}\right)\right]$. Then $F$ satisfies all the conditions of Theorem 3.1, but not covered by the results of $[1-15]$.

For example, let $x=\left(x_{1}, x_{2}, \ldots, x_{N}\right)^{T} \in \mathbb{R}^{N}, \omega(|x|)=|x|$ and

$$
\begin{aligned}
F(t, x)= & \left(\frac{5}{6} T-t\right)\left(r+1+\sum_{j=1}^{r} \sin ^{2} x_{j}+\frac{1}{2} \sum_{j=r+1}^{N} x_{j}^{2}\right) \\
& +\left(T^{3}-t\right)\left(r+1+\sum_{j=1}^{r} \sin ^{2} x_{j}+\frac{1}{2} \sum_{j=r+1}^{N} x_{j}^{2}\right)^{\frac{1}{2}},
\end{aligned}
$$

where $1 \leq r \leq m$. Then $F$ satisfies all the conditions of Theorem 3.2, but not covered by the results of [1-15].

For the sake of convenience, we denote by $C_{i}(i=1,2,3, \ldots, 33)$ various positive constants.

Proof of Theorem 3.1 First, we prove that $\psi$ satisfies the (PS) condition. Let $\pi: W_{T}^{1, p(t)} \rightarrow$ $W_{T}^{1, p(t)} / G$ be the canonical surjection. Define $\psi: X \times V \mapsto \mathbb{R}$ by $\psi(\pi(u))=\varphi(u)$. Assume that $\left(\pi\left(u_{n}\right)\right)$ is a (PS) sequence for $\psi$, that is, $\psi\left(\pi\left(u_{n}\right)\right)$ is bounded and $\psi^{\prime}\left(\pi\left(u_{n}\right)\right) \rightarrow 0$. Then $\varphi\left(u_{n}\right)$ is bounded and $\varphi^{\prime}\left(u_{n}\right) \rightarrow 0$.

We can get from $(\omega 1),(\omega 2)$, and $(\omega 3)$ that

$$
\begin{aligned}
\omega(|\hat{u}(t)|)= & \omega\left(\left|u^{+}(t)+u^{-}(t)+Q \hat{u}^{0}+P u^{0}\right|\right) \\
\leq & \omega\left(\left|u^{+}\right|+\left|u^{-}\right|+\left|Q \hat{u}^{0}\right|+\left|P u^{0}\right|\right) \\
\leq & M_{0}\left[\omega\left(\left|u^{+}\right|+\left|u^{-}\right|+\left|Q \hat{u}^{0}\right|\right)+\omega\left(\left|P u^{0}\right|\right)\right] \\
\leq & M_{0}\left[M_{1}\left(\left|u^{+}\right|+\left|u^{-}\right|+\left|Q \hat{u}^{0}\right|\right)+M_{2}\right]+M_{0} \omega\left(\left|P u^{0}\right|\right) \\
\leq & M_{0} M_{1}\left(\left\|u^{+}\right\|_{\infty}+\left\|u^{-}\right\|_{\infty}\right)+M_{0} M_{1}\left|Q \hat{u}^{0}\right|+M_{0} M_{2} \\
& +M_{0} \omega\left(\left|P u^{0}\right|\right) .
\end{aligned}
$$

By (2.3) and the boundedness of $\left|Q \hat{u}^{0}\right|$, we have

$$
\begin{aligned}
& \left|\int_{0}^{T}\left(\nabla F(t, \hat{u}(t)), u^{+}(t)\right) d t\right| \\
& \quad \leq \int_{0}^{T} f(t) \omega(|\hat{u}(t)|)\left|u^{+}(t)\right| d t+\int_{0}^{T} g(t)\left|u^{+}(t)\right| d t
\end{aligned}
$$




$$
\begin{aligned}
\leq & M_{0} M_{1} \int_{0}^{T} f(t) d t\left(\left\|u^{+}\right\|_{\infty}^{2}+\left\|u^{-}\right\|_{\infty}\left\|u^{+}\right\|_{\infty}\right) \\
& +M_{0} \int_{0}^{T} f(t) d t \omega\left(\left|P u^{0}\right|\right)\left\|u^{+}\right\|_{\infty}+C_{1}\left\|u^{+}\right\|_{\infty} \\
\leq & M_{0} M_{1} C_{0}^{2} \int_{0}^{T} f(t) d t\left(\left\|u^{+}\right\|^{2}+\left\|u^{-}\right\|\left\|u^{+}\right\|\right) \\
& +M_{0} C_{0} \int_{0}^{T} f(t) d t \omega\left(\left|P u^{0}\right|\right)\left\|u^{+}\right\|+C_{2}\left\|u^{+}\right\| \\
\leq & M_{0} M_{1} C_{0}^{2} \int_{0}^{T} f(t) d t\left(\left\|u^{+}\right\|^{2}+\frac{\left\|u^{-}\right\|^{2}+\left\|u^{+}\right\|^{2}}{2}\right) \\
& +M_{0} C_{0} \int_{0}^{T} f(t) d t \frac{\frac{\omega^{2}\left(\left|P u^{0}\right|\right)}{M_{1} C_{0}}+M_{1} C_{0}\left\|u^{+}\right\|^{2}}{2}+C_{2}\left\|u^{+}\right\| \\
= & M_{0} M_{1} C_{0}^{2} \int_{0}^{T} f(t) d t\left(2\left\|u^{+}\right\|^{2}+\frac{1}{2}\left\|u^{-}\right\|^{2}\right) \\
& +\frac{1}{2} \frac{M_{0}}{M_{1}} \int_{0}^{T} f(t) d t \omega^{2}\left(\left|P u^{0}\right|\right)+C_{2}\left\|u^{+}\right\|
\end{aligned}
$$

From (H3) and (2.3), we obtain that

$$
\begin{aligned}
\left|\int_{0}^{T}\left(h(t), u^{+}(t)\right) d t\right| & \leq\left\|u^{+}\right\|_{\infty} \int_{0}^{T}|h(t)| d t \\
& \leq C_{0}\left\|u^{+}\right\| \int_{0}^{T}|h(t)| d t .
\end{aligned}
$$

It follows from (2.2), (3.5), and (3.6) that

$$
\begin{aligned}
\left\|u_{n}^{+}\right\| \geq & \left\langle\varphi^{\prime}\left(u_{n}\right), u_{n}^{+}\right\rangle \\
= & \left\langle\varphi^{\prime}\left(\hat{u}_{n}\right), u_{n}^{+}\right\rangle \\
= & \int_{0}^{T}\left|\dot{u}_{n}^{+}(t)\right|^{2} d t-\int_{0}^{T}\left(A u_{n}(t), u_{n}^{+}(t)\right) d t \\
& +\int_{0}^{T}\left(\nabla F\left(t, \hat{u}_{n}(t)\right), u_{n}^{+}(t)\right) d t+\int_{0}^{T}\left(h(t), u_{n}^{+}(t)\right) d t \\
\geq & \left(\delta-2 M_{0} M_{1} C_{0}^{2} \int_{0}^{T} f(t) d t\right)\left\|u_{n}^{+}\right\|^{2} \\
& -\frac{1}{2} M_{0} M_{1} C_{0}^{2} \int_{0}^{T} f(t) d t\left\|u_{n}^{-}\right\|^{2} \\
& -\frac{1}{2} \frac{M_{0}}{M_{1}} \int_{0}^{T} f(t) d t \omega^{2}\left(\left|P u_{n}^{0}\right|\right)+C_{3}\left\|u_{n}^{+}\right\|
\end{aligned}
$$

for large $n$. So we have

$$
\begin{aligned}
& \frac{1}{2} M_{0} M_{1} C_{0}^{2} \int_{0}^{T} f(t) d t\left(\left\|u_{n}^{-}\right\|^{2}+\frac{\omega^{2}\left(\left|P u_{n}^{0}\right|\right)}{M_{1}^{2} C_{0}^{2}}\right) \\
& \quad \geq\left(\delta-2 M_{0} M_{1} C_{0}^{2} \int_{0}^{T} f(t) d t\right)\left\|u_{n}^{+}\right\|^{2}-C_{4}\left\|u_{n}^{+}\right\|
\end{aligned}
$$


Chang Boundary Value Problems ( 2017) 2017:99

Page 9 of 14

$$
\begin{aligned}
= & {\left[\delta-(2+a) M_{0} M_{1} C_{0}^{2} \int_{0}^{T} f(t) d t\right]\left\|u_{n}^{+}\right\|^{2} } \\
& +a M_{0} M_{1} C_{0}^{2} \int_{0}^{T} f(t) d t\left\|u_{n}^{+}\right\|^{2}-C_{4}\left\|u_{n}^{+}\right\| \\
\geq & a M_{0} M_{1} C_{0}^{2} \int_{0}^{T} f(t) d t\left\|u_{n}^{+}\right\|^{2}+C_{5},
\end{aligned}
$$

where $C_{5}=\min _{s \in[0,+\infty)}\left\{\left[\delta-(2+a) M_{0} M_{1} C_{0}^{2} \int_{0}^{T} f(t) d t\right] s^{2}-C_{4} s\right\}$.

From (3.1), one has that $(2+a) M_{0} M_{1} C_{0}^{2} \int_{0}^{T} f(t) d t<\delta$, then $C_{5}<0$. Hence

$$
\left\|u_{n}^{+}\right\|^{2} \leq \frac{1}{2 a}\left(\left\|u_{n}^{-}\right\|^{2}+\frac{\omega^{2}\left(\left|P u_{n}^{0}\right|\right)}{M_{1}^{2} C_{0}^{2}}\right)+C_{6} .
$$

In a similar way, we have

$$
\left\|u_{n}^{-}\right\|^{2} \leq \frac{1}{2 a}\left(\left\|u_{n}^{+}\right\|^{2}+\frac{\omega^{2}\left(\left|P u_{n}^{0}\right|\right)}{M_{1}^{2} C_{0}^{2}}\right)+C_{7}
$$

Combining the above two inequalities, one has that

$$
\begin{aligned}
\left\|u_{n}^{+}\right\|^{2} & \leq \frac{1}{2 a}\left(\left\|u_{n}^{-}\right\|^{2}+\frac{\omega^{2}\left(\left|P u_{n}^{0}\right|\right)}{M_{1}^{2} C_{0}^{2}}\right)+C_{6} \\
& \leq \frac{1}{4 a^{2}}\left\|u_{n}^{+}\right\|^{2}+\frac{1}{2 a}\left(\frac{1}{2 a}+1\right) \frac{\omega^{2}\left(\left|P u_{n}^{0}\right|\right)}{M_{1}^{2} C_{0}^{2}}+\frac{C_{7}}{2 a}+C_{6} .
\end{aligned}
$$

Consequently,

$$
\left\|u_{n}^{+}\right\|^{2} \leq \frac{1}{2 a-1} \frac{\omega^{2}\left(\left|P u_{n}^{0}\right|\right)}{M_{1}^{2} C_{0}^{2}}+C_{8} .
$$

Using similar arguments, we can prove that

$$
\left\|u_{n}^{-}\right\|^{2} \leq \frac{1}{2 a-1} \frac{\omega^{2}\left(\left|P u_{n}^{0}\right|\right)}{M_{1}^{2} C_{0}^{2}}+C_{9} .
$$

By (3.5), (3.6), (3.7), (3.8), and (3.9), we have

$$
\begin{aligned}
& \int_{0}^{T}\left|\dot{u}_{n}^{+}(t)\right|^{2} d t-\int_{0}^{T}\left(A u_{n}(t), u_{n}^{+}(t)\right) d t \\
& \leq\left\|u_{n}^{+}\right\|-\int_{0}^{T}\left(\nabla F\left(t, \hat{u}_{n}(t)\right), u_{n}^{+}(t)\right) d t \\
& \quad-\int_{0}^{T}\left(h(t), u_{n}^{+}(t)\right) d t \\
& \leq M_{0} M_{1} C_{0}^{2} \int_{0}^{T} f(t) d t\left(2\left\|u_{n}^{+}\right\|^{2}+\frac{1}{2}\left\|u_{n}^{-}\right\|^{2}\right) \\
& \quad+\frac{1}{2} \frac{M_{0}}{M_{1}} \int_{0}^{T} f(t) d t \omega^{2}\left(\left|P u^{0}\right|\right)+C_{10}\left\|u_{n}^{+}\right\|
\end{aligned}
$$




$$
\begin{aligned}
\leq & \left(\frac{5}{2} \frac{1}{2 a-1}+\frac{1}{2}\right) \frac{M_{0}}{M_{1}} \int_{0}^{T} f(t) d t \omega^{2}\left(\left|P u_{n}^{0}\right|\right) \\
& +C_{11} \omega\left(\left|P u_{n}^{0}\right|\right)+C_{12} .
\end{aligned}
$$

In a similar way, we can obtain

$$
\begin{aligned}
& \int_{0}^{T}\left|\dot{u}_{n}^{-}(t)\right|^{2} d t-\int_{0}^{T}\left(A u_{n}(t), u_{n}^{-}(t)\right) d t \\
& \leq\left(\frac{5}{2} \frac{1}{2 a-1}+\frac{1}{2}\right) \frac{M_{0}}{M_{1}} \int_{0}^{T} f(t) d t \omega^{2}\left(\left|P u_{n}^{0}\right|\right) \\
& \quad+C_{13} \omega\left(\left|P u_{n}^{0}\right|\right)+C_{14} .
\end{aligned}
$$

By (3.2) and ( $\omega 1)$, one has

$$
\begin{aligned}
& \left|\int_{0}^{T} F\left(t, \hat{u}_{n}(t)\right) d t-\int_{0}^{T} F\left(t, P u_{n}^{0}\right) d t\right| \\
& \quad=\left|\int_{0}^{T} \int_{0}^{1}\left(\nabla F\left(t, P u_{n}^{0}+s\left(Q \hat{u}_{n}^{0}+u_{n}^{+}+u_{n}^{-}\right)\right), Q \hat{u}_{n}^{0}+u_{n}^{+}+u_{n}^{-}\right) d s d t\right| \\
& \quad \leq \int_{0}^{T} \int_{0}^{1} f(t) \omega\left(\left|P u_{n}^{0}\right|+\left|Q \hat{u}_{n}^{0}\right|+\left|u_{n}^{+}\right|+\left|u_{n}^{-}\right|\right)\left(\left|Q \hat{u}_{n}^{0}\right|+\left|u_{n}^{+}\right|+\left|u_{n}^{-}\right|\right) d s d t \\
& \quad+\int_{0}^{T} \int_{0}^{1} g(t)\left(\left|Q \hat{u}_{n}^{0}\right|+\left|u_{n}^{+}\right|+\left|u_{n}^{-}\right|\right) d s d t .
\end{aligned}
$$

From (3.4), (2.3), and the boundedness of $\left|Q \hat{u}^{0}\right|$, we have

$$
\begin{aligned}
& \left|\int_{0}^{T} F\left(t, \hat{u}_{n}(t)\right) d t-\int_{0}^{T} F\left(t, P u_{n}^{0}\right) d t\right| \\
& \leq \int_{0}^{T} f(t) d t M_{0} M_{1}\left(\left|Q \hat{u}_{n}^{0}\right|+\left\|u_{n}^{+}\right\|_{\infty}+\left\|u_{n}^{-}\right\|_{\infty}\right)^{2} \\
& \quad+\int_{0}^{T} f(t) d t M_{0} M_{2}\left(\left|Q \hat{u}_{n}^{0}\right|+\left\|u_{n}^{+}\right\|_{\infty}+\left\|u_{n}^{-}\right\|_{\infty}\right) \\
& \quad+\int_{0}^{T} f(t) d t M_{0} \omega\left(\left|P u_{n}^{0}\right|\right)\left(\left|Q \hat{u}_{n}^{0}\right|+\left\|u_{n}^{+}\right\|_{\infty}+\left\|u_{n}^{-}\right\|_{\infty}\right) \\
& \quad+\int_{0}^{T} g(t) d t\left(\left|Q \hat{u}_{n}^{0}\right|+\left\|u_{n}^{+}\right\|_{\infty}+\left\|u_{n}^{-}\right\|_{\infty}\right) \\
& \leq M_{0} M_{1} C_{0}^{2} \int_{0}^{T} f(t) d t\left(\left\|u_{n}^{+}\right\|+\left\|u_{n}^{-}\right\|\right)^{2} \\
& \quad+M_{0} C_{0} \int_{0}^{T} f(t) d t \omega\left(\left|P u_{n}^{0}\right|\right)\left(\left\|u_{n}^{+}\right\|+\left\|u_{n}^{-}\right\|\right) \\
& \quad+C_{17}\left(\left\|u_{n}^{+}\right\|+\left\|u_{n}^{-}\right\|\right)+C_{18} \omega\left(\left|P u_{n}^{0}\right|\right)+C_{19} .
\end{aligned}
$$

Hence, we have

$$
\begin{aligned}
& \left|\int_{0}^{T} F\left(t, \hat{u}_{n}(t)\right) d t-\int_{0}^{T} F\left(t, P u_{n}^{0}\right) d t\right| \\
& \quad \leq M_{0} M_{1} C_{0}^{2} \int_{0}^{T} f(t) d t 2\left(\left\|u_{n}^{+}\right\|^{2}+\left\|u_{n}^{-}\right\|^{2}\right)
\end{aligned}
$$




$$
\begin{aligned}
& +M_{0} C_{0} \int_{0}^{T} f(t) d t \frac{\frac{1}{M_{1} C_{0}} \omega^{2}\left(\left|P u_{n}^{0}\right|\right)+M_{1} C_{0}\left\|u_{n}^{+}\right\|^{2}}{2} \\
& +M_{0} C_{0} \int_{0}^{T} f(t) d t \frac{\frac{1}{M_{1} C_{0}} \omega^{2}\left(\left|P u_{n}^{0}\right|\right)+M_{1} C_{0}\left\|u_{n}^{-}\right\|^{2}}{2} \\
& +C_{17}\left(\left\|u_{n}^{+}\right\|+\left\|u_{n}^{-}\right\|\right)+C_{18} \omega\left(\left|P u_{n}^{0}\right|\right)+C_{19} \\
& \leq \frac{5}{2} M_{0} M_{1} C_{0}^{2} \int_{0}^{T} f(t) d t\left(\left\|u_{n}^{+}\right\|^{2}+\left\|u_{n}^{-}\right\|^{2}\right) \\
& +\frac{M_{0}}{M_{1}} \int_{0}^{T} f(t) d t \omega^{2}\left(\left|P u_{n}^{0}\right|\right) \\
& +C_{17}\left(\left\|u_{n}^{+}\right\|+\left\|u_{n}^{-}\right\|\right)+C_{18} \omega\left(\left|P u_{n}^{0}\right|\right)+C_{19} .
\end{aligned}
$$

By (3.8), (3.9), and (3.12), we have

$$
\begin{aligned}
& \left|\int_{0}^{T} F\left(t, \hat{u}_{n}(t)\right) d t-\int_{0}^{T} F\left(t, P u_{n}^{0}\right) d t\right| \\
& \quad \leq\left(\frac{5}{2 a-1}+1\right) \frac{M_{0}}{M_{1}} \int_{0}^{T} f(t) d t \omega^{2}\left(\left|P u_{n}^{0}\right|\right) \\
& \quad+C_{20} \omega\left(\left|P u_{n}^{0}\right|\right)+C_{21} .
\end{aligned}
$$

From (H3), (2.3), (3.8), and (3.9), one has

$$
\begin{aligned}
\left|\int_{0}^{T}\left(h(t), \hat{u}_{n}(t)\right) d t\right| & \leq \int_{0}^{T}|h(t)| d t\left(\left\|u_{n}^{+}\right\|_{\infty}+\left\|u_{n}^{-}\right\|_{\infty}\right) \\
& \leq C_{0} \int_{0}^{T}|h(t)| d t\left(\left\|u_{n}^{+}\right\|+\left\|u_{n}^{-}\right\|\right) \\
& \leq C_{15} \omega\left(\left|P u_{n}^{0}\right|\right)+C_{16} .
\end{aligned}
$$

It follows from (3.10), (3.11), (3.13), and (3.14) that

$$
\begin{aligned}
\varphi\left(u_{n}\right)= & \varphi\left(\hat{u}_{n}\right) \\
= & \frac{1}{2}\left[\int_{0}^{T}\left|\dot{u}_{n}^{+}(t)\right|^{2} d t-\int_{0}^{T}\left(A u_{n}(t), u_{n}^{+}(t)\right) d t\right] \\
& +\frac{1}{2}\left[\int_{0}^{T}\left|\dot{u}_{n}^{-}(t)\right|^{2} d t-\int_{0}^{T}\left(A u_{n}(t), u_{n}^{-}(t)\right) d t\right] \\
& +\left[\int_{0}^{T} F\left(t, \hat{u}_{n}(t)\right) d t-\int_{0}^{T} F\left(t, P u_{n}^{0}\right) d t\right] \\
& +\int_{0}^{T} F\left(t, P u_{n}^{0}\right) d t+\int_{0}^{T}\left(h(t), \hat{u}_{n}(t)\right) d t \\
\leq & {\left[\left(\frac{15}{2} \frac{1}{2 a-1}+\frac{3}{2}\right) \frac{M_{0}}{M_{1}} \int_{0}^{T} f(t) d t+\frac{\int_{0}^{T} F\left(t, P u_{n}^{0}\right) d t}{\omega^{2}\left(\left|P u_{n}^{0}\right|\right)}\right] \omega^{2}\left(\left|P u_{n}^{0}\right|\right) } \\
& +C_{22} \omega\left(\left|P u_{n}^{0}\right|\right)+C_{23},
\end{aligned}
$$


which implies $\left|P u_{n}^{0}\right|$ is bounded. Otherwise, we assume $\left|P u_{n}^{0}\right| \rightarrow \infty$ as $n \rightarrow \infty$. From ( $\left.\omega 4\right)$, we obtain that

$$
\omega\left(\left|P u_{n}^{0}\right|\right) \rightarrow+\infty \quad \text { as } n \rightarrow \infty
$$

By (3.3), we conclude that

$$
\varphi\left(u_{n}\right) \rightarrow-\infty \quad \text { as } n \rightarrow \infty,
$$

this contradicts the boundedness of $\left\{\varphi\left(u_{n}\right)\right\}$, so $\left|P u_{n}^{0}\right|$ is bounded. Combining (3.8) and (3.9), we obtain that $\left\|u_{n}^{+}\right\|$and $\left\|u_{n}^{-}\right\|$are bounded. Furthermore, $\left|Q \hat{u}^{0}\right|$ is bounded, so $\left\{\hat{u}_{n}\right\}$ is bounded in $H_{T}^{1}$. Arguing then as in Proposition 4.1 in [6], $\left\{\hat{u}_{n}\right\}$ has a convergent subsequence. By $\pi\left(\hat{u}_{n}\right)=\pi\left(u_{n}\right)$, we conclude that $\psi$ satisfies the (PS) condition.

Next, we only need to verify the linking conditions of the generalized saddle point theorem:

(a) For $\pi(u) \in W \times V, u(t)=u^{+}(t)+Q u^{0}$. By the proof of (3.12), we have

$$
\begin{aligned}
& \left|\int_{0}^{T} F\left(t, u^{+}(t)+Q u^{0}\right) d t-\int_{0}^{T} F(t, 0) d t\right| \\
& \quad \leq \frac{5}{2} M_{0} M_{1} C_{0}^{2} \int_{0}^{T} f(t) d t\left\|u^{+}\right\|^{2}+C_{24}\left\|u^{+}\right\|+C_{25} .
\end{aligned}
$$

Hence

$$
\begin{aligned}
\psi(\pi(u))= & \psi\left(\pi\left(u^{+}+Q u^{0}\right)\right) \\
= & \varphi\left(u^{+}+Q u^{0}\right) \\
= & \frac{1}{2} \int_{0}^{T}\left|\dot{u}^{+}(t)\right|^{2} d t+\frac{1}{2} \int_{0}^{T}\left(A u(t), u^{+}(t)\right) d t \\
& +\left[\int_{0}^{T} F\left(t, u^{+}(t)+Q u^{0}\right) d t-\int_{0}^{T} F(t, 0) d t\right] \\
& +\int_{0}^{T}\left(h(t), u^{+}(t)+Q u^{0}\right) d t+\int_{0}^{T} F(t, 0) d t \\
\geq & \left(\frac{\delta}{2}-\frac{5}{2} M_{0} M_{1} C_{0}^{2} \int_{0}^{T} f(t) d t\right)\left\|u^{+}\right\|^{2}-C_{26}\left\|u^{+}\right\|-C_{27} .
\end{aligned}
$$

Note the boundedness of $\left|Q u^{0}\right|$ and (3.1), we obtain that $\psi(\pi(u)) \rightarrow+\infty$ as $\|u\| \rightarrow-\infty$ for all $\pi(u) \in W \times V$, which implies that there exists $\beta \in \mathbb{R}$ such that $\psi(\pi(u)) \geq \beta$ on $W \times V$.

(b) In a way similar to the proof of (3.12), we have

$$
\begin{aligned}
& \left|\int_{0}^{T} F\left(t, u^{-}+P u^{0}+Q u^{0}\right) d t-\int_{0}^{T} F\left(t, P u^{0}\right) d t\right| \\
& \quad \leq \frac{5}{2} M_{0} M_{1} C_{0}^{2} \int_{0}^{T} f(t) d t\left\|u^{-}\right\|^{2}+\frac{M_{0}}{M_{1}} \int_{0}^{T} f(t) d t \omega^{2}\left(\left|P u^{0}\right|\right) \\
& \quad+C_{28}\left\|u^{-}\right\|+C_{29} \omega\left(\left|P u^{0}\right|\right)+C_{30} .
\end{aligned}
$$


Consequently,

$$
\begin{aligned}
\psi(\pi(u))= & \psi\left(\pi\left(u^{-}+P u^{0}+Q u^{0}\right)\right) \\
= & \varphi\left(u^{-}+P u^{0}+Q u^{0}\right) \\
= & \frac{1}{2} \int_{0}^{T}\left|\dot{u}^{-}\right|^{2} d t+\frac{1}{2} \int_{0}^{T}\left(A u, u^{-}\right) d t+\int_{0}^{T} F\left(t, P u^{0}\right) d t \\
& +\left[\int_{0}^{T} F\left(t, u^{-}+P u^{0}+Q u^{0}\right) d t-\int_{0}^{T} F\left(t, P u^{0}\right) d t\right] \\
& +\int_{0}^{T}\left(h(t), u^{-}+P u^{0}+Q u^{0}\right) d t \\
\leq & \frac{1}{2}\left[-\delta+5 M_{0} M_{1} C_{0}^{2} \int_{0}^{T} f(t) d t\right]\left\|u^{-}\right\|^{2}+C_{31}\left\|u^{-}\right\| \\
& +\left[\frac{M_{0}}{M_{1}} \int_{0}^{T} f(t) d t+\frac{\int_{0}^{T} F\left(t, P u^{0}\right) d t}{\omega^{2}\left(\left|P u^{0}\right|\right)}\right] \omega^{2}\left(\left|P u^{0}\right|\right) \\
& +C_{32} \omega\left(\left|P u^{0}\right|\right)+C_{33} .
\end{aligned}
$$

From $a>3$ and (3.1), one has that $-\delta+5 M_{0} M_{1} C_{0}^{2} \int_{0}^{T} f(t) d t<0$.

By (3.3), we deduce that

$$
\underset{\left|P u^{0}\right| \rightarrow \infty}{\limsup } \frac{\int_{0}^{T} F\left(t, P u^{0}\right) d t}{\omega^{2}\left(\left|P u^{0}\right|\right)}<-\frac{M_{0}}{M_{1}} \int_{0}^{T} f(t) d t,
$$

so we obtain that $\psi(\pi(u)) \rightarrow+\infty$ as $\|u\| \rightarrow-\infty$ for all $\pi(u) \in Z \times V$, which implies that there exists $\gamma<\beta$ such that $\psi(\pi(u)) \leq \gamma$ on $Z \times V$.

The functional $\psi$ satisfies all the assumptions of Lemma 2.2, so it has at least cuplength $(V)+1$ critical points, and since $V$ is the torus $T^{r}$, then cuplength $(V)=r$. Hence $\varphi$ has at least $r+1$ critical points. Therefore, problem (1.1) has at least $r+1$ distinct solutions in $H_{T}^{1}$. The proof of Theorem 3.1 is completed.

Proof of Theorem 3.2 The proof of Theorem 3.2 is similar to the proof of Theorem 3.1, so we omit the discussions here.

Remark 3.2 Using the parallel arguments with little change as in the proofs of Theorems 3.1 and 3.2, the conclusions of Theorems 3.1 and 3.2 hold if we replace $(\omega 3)$ with

$(\omega 3)^{\prime} \quad 0 \leq \omega(s) \leq M_{1} s^{\alpha}+M_{2}, \forall s, t \in[0, \infty)$, where $0 \leq \alpha<1$.

\section{Acknowledgements}

This work is supported by the National Natural Science Foundation of China (No. 31260098).

\section{Competing interests}

The author declares that they have no competing interests.

Author's contributions

The author read and approved the final manuscript.

\section{Publisher's Note}

Springer Nature remains neutral with regard to jurisdictional claims in published maps and institutional affiliations. 
Received: 26 September 2016 Accepted: 3 May 2017 Published online: 05 July 2017

\section{References}

1. Chang, KQ: On the periodic nonlinearity and the multiplicity of solutions. Nonlinear Anal., Theory Methods Appl. 13, 527-537 (1989)

2. Faraci, F: Multiple periodic solutions for second order systems with changing sign potential. J. Math. Anal. Appl. 319, 567-578 (2006)

3. Feng, JX, Han, ZQ: Periodic solutions to differential systems with unbounded or periodic nonlinearities. J. Math. Anal. Appl. 323, 1264-1278 (2006)

4. Han, ZQ, Wang, SQ: Multiple solutions for nonlinear systems with gyroscopic terms. Nonlinear Anal., Theory Methods Appl. 75, 5756-5764 (2012)

5. Liu, JQ: A generalized saddle point theorem. J. Differ. Equ. 82, 372-385 (1989)

6. Mawhin, J, Willem, M: Critical Point Theory and Hamiltonian Systems. Springer, New York (1989)

7. Ning, Y, An, TQ: Periodic solutions of a class of nonautonomous second-order Hamiltonian systems with nonsmooth potentials. Bound. Value Probl. 2015, 34 (2015)

8. Pipan, J, Schechter, M: Non-autonomous second order Hamiltonian systems. J. Differ. Equ. 257, 351-373 (2014)

9. Schechter, M: Periodic second order superlinear Hamiltonian systems. J. Math. Anal. Appl. 426, 546-562 (2015)

10. Wang, ZY, Zhang, JH: Periodic solutions of a class of second order non-autonomous Hamiltonian systems. Nonlinear Anal., Theory Methods Appl. 72, 4480-4487 (2010)

11. Xiao, L: Existence of periodic solutions for second order Hamiltonian system. Bull. Malays. Math. Soc. 35, 785-801 (2012)

12. Tang, CL: Periodic solutions of second order nonautonomous systems with sublinear nonlinearity. Proc. Am. Math. Soc. 126, 3263-3270 (1998)

13. Tang, CL: A note on periodic solutions of second order systems. Proc. Am. Math. Soc. 132, 1295-1393 (2003)

14. Zhang, XY, Tang, XH: Periodic solutions for an ordinary p-Laplacian system. Taiwan. J. Math. 12, 1369-1396 (2011)

15. Zhang, SG: Multiple periodic solutions for a class of sublinear nonautonomous second order system. Acta Anal. Funct. Appl. 15, 12-20 (2013)

16. Rabinowitz, PH: Minimax Methods in Critical Point Theory with Applications to Differential Equations. CBMS Regional Conference Series in Mathematics, vol. 65. Am. Math. Soc., Providence (1986)

17. Willem, M: Minimax Theorems. Birkhäuser, Boston (1996)

18. Schechter, M: Linking Methods in Critical Point Theory. Birkhäuser, Boston (1999)

19. Bartsch, T: Critical point theory on partially ordered Hilbert spaces. J. Funct. Anal. 186, 117-152 (2001)

20. Shahmurov, R: Solution of the Dirichlet and Neumann problems for a modified Helmholtz equation in Besov spaces on an annulus. J. Differ. Equ. 249, 526-550 (2010)

\section{Submit your manuscript to a SpringerOpen ${ }^{\circ}$ journal and benefit from:}

- Convenient online submission

- Rigorous peer review

- Open access: articles freely available online

- High visibility within the field

- Retaining the copyright to your article

Submit your next manuscript at $\boldsymbol{~ s p r i n g e r o p e n . c o m ~}$ 